\title{
Calpain inhibitors ameliorate muscle wasting in a cachectic mouse model bearing CT26 colorectal adenocarcinoma
}

\author{
XING-YU LIN ${ }^{1}$ and SI-ZENG CHEN ${ }^{1,2}$ \\ ${ }^{1}$ The First Clinical Medical College of Fujian Medical University; ${ }^{2}$ Department of Gastrointestinal Surgery, \\ The First Affiliated Hospital, Fujian Medical University, Fuzhou, Fujian 350005, P.R. China
}

Received August 21, 2016; Accepted January 5, 2017

DOI: 10.3892/or.2017.5396

\begin{abstract}
Cancer-related cachexia involves increased protein breakdown through various proteolytic pathways, including the ubiquitin-proteasome pathway (UPP). We hypothesized that a calcium- and calpain-dependent pathway might play a crucial role during the proteolytic procedure, and that pathway interventions would ameliorate cancer cachexia in vivo. After being inoculated with CT26 adenocarcinoma cell culture subcutaneously, BALB/c mice developed cachexia in 12 days. They were then administered with different types of calpain inhibitors individually or in combination for 7 consecutive days. Eighteen healthy mice were also assessed as a control group. Changes in body weight, gastrocnemius muscle mass, tumor volume, food intake, survival time, and serum nutritional markers were monitored. Also measured were the levels of calpains, E3 ubiquitin ligases, and apoptosis-associated markers in gastrocnemius muscle. Our study showed that the intraperitoneal administration of calpain inhibitors significantly improved tumor-free body weight and gastrocnemius muscle mass in all treatment groups. Treatment with calpain inhibitors also ameliorated cachexia-associated negative effects in metabolic profiles and increased survival time in most of the tumor-bearing mice compared with the cachexia controls. Furthermore, calpain inhibitors reduced the calpain activity and the expression of MuRF-1 and atrogin-1 in all
\end{abstract}

Correspondence to: Dr Si-Zeng Chen, Department of Gastrointestinal Surgery, The First Affiliated Hospital, Fujian Medical University, 20 Chating Zhong Road, Fuzhou, Fujian 350005, P.R. China

E-mail: chensz04871@hotmail.com

Abbreviations: UPP, ubiquitin-proteasome proteolysis; CAST, calpastatin; IV, calpain inhibitor IV; DMSO, dimethyl sulfoxide; PCR, polymerase chain reaction; qRT-PCR: quantitative real-time polymerase chain reaction; cDNA, complementary deoxyribonucleic acid; MAFbx, muscle atrophy F-box; MuRF-1, muscle ring finger-1; GAPDH, glyceraldehyde-3-phosphate dehydrogenase; BAX, BCL2associated X; BCL-2: B-cell lymphoma/leukemia-2

Key words: cancer cachexia, calpain inhibitor, muscle wasting, proteolysis, apoptosis treatment groups, while increasing the level of cleaved caspase-3 and BAX and lowering the level of BCL-2 in some groups. These results justify further evaluation of calpain inhibitors both alone and in combination with other candidate agents as a potential new therapeutic strategy for treating cancer cachexia.

\section{Introduction}

Cancer cachexia is a multifactorial syndrome characterized by a progressive loss of skeletal muscle mass that cannot be fully compensated for by conventional treatments such as increasing energy intake through nutritional support (1). Approximately $60-80 \%$ of all advanced cancer patients experience cachexia, particularly those with gastric and pancreatic cancers (2). Cachexia is associated with decreased physical function, impaired quality of life, and shortened survival time (3), and is responsible for roughly $20 \%$ of all cancer deaths (4). With growing incidence and high mortality, it also increases health care resource utilization and associated hospitalization costs, and has become a major social health problem (5). Since a significant unmet need exists for improving patients' quality of life and decreasing the public health burden, it is critical to clarify the mechanism of this debilitating condition.

As the most important feature of cancer cachexia, skeletal muscle atrophy is believed to be due to a significant increase in proteolysis of myofibrils (6). Although mounting evidence indicates that the ubiquitin-proteasome pathway (UPP) plays a prominent role in the process $(7,8)$, the proteasome only degrades peptides by engulfing them in its catalytic chamber and cannot disassemble myofibrillar proteins until they have been released from the myofibrils as myofilaments by the calpain system (6). Furthermore, evaluation of muscle biopsies in clinical trials suggests that muscle apoptosis exists in this hypercatabolic state, which contributes to the severity of the cachexia (9).

Calpain, a member of the calcium-activated cysteine protease family, is implicated in diverse diseases, including muscular dystrophy, neurological disorders, cataracts, and hematonosis (10). Inhibition of calpain can preserve the function of critical proteins by suppressing associated proteolysis, thus potentially ameliorating these diseases. In terms of cachexia-induced muscle wasting, calpain and UPP have a synergistic effect on muscle protein proteolysis, as calpains 
can provide substrates for UPP when activated (11). In addition, calpain inhibitors prevent calpain-mediated neuron apoptosis in several rodent models of nervous system injury $(12,13)$. These inhibitors thus might also protect skeletal muscle from cachexia-induced apoptosis.

In this study, we evaluated the effect of three common calpain inhibitors, calpastatin (an endogenous polypeptide inhibitor), calpain inhibitor IV, and calpeptin (both irreversible inhibitors derived from chemical synthesis) on skeletal muscle proteolysis and apoptosis in a murine model of cancer cachexia.

\section{Materials and methods}

Reagents and cell line. Calpastatin peptide (CAST for short), calpain inhibitor IV (IV for short) and Calpeptin (Merck Millipore, Boston, MA, USA, catalogue no. 208902, 208724, 03-34-0051) were resolved with dimethyl sulfoxide (DMSO) as a stock solution and diluted in sterile saline solution before use. Primary antibodies [rabbit monoclonal anti-calpain 1 (ab108400; 1:1000); rabbit monoclonal anti-calpain 2 (ab126600; 1:1000); rabbit polyclonal anti-calpastatin (ab28252; 1:5000); rabbit monoclonal anti-Bax (ab32503; 1:1000); rabbit polyclonal anti-Bcl-2 (ab59348; 1:1000); mouse monoclonal anti-GAPDH (ab9484; 1:1000)] were purchased from Abcam (Cambridge, UK), rabbit polyclonal anti-cleaved caspase-3 (Asp175) (cat\# 9661; 1:1000) was obtained from Cell Signaling Technology (Danvers, MA, USA), and secondary goat anti-rabbit IgG (BA1055; 1:3000) and goat anti-mouse IgG (BA1050; 1:3000) conjugated with horseradish peroxidase were obtained from Boster (Wuhan, China). Colon carcinoma cell line CT26 was purchased from American Type Culture Collection (Manassas, VA, USA).

Design of animal experiments. BALB/c male mice (6-8 weeks old, body weight 20-24 g) were purchased from the Animal Center of the Chinese Academy of Sciences (Shanghai, China; Security processing facility certificate number SCXK (Hu) 2012-0002). The animals were maintained in a specific pathogen-free environment at a controlled temperature $\left(22 \pm 1^{\circ} \mathrm{C}\right)$ and a $12 \mathrm{~h}$ light-dark cycle, which was provided by the Animal Experiments Center of Fujian Medical University. All animals were fed with standard laboratory chow and tap ad libitum. The protocol was approved by the Institutional Animal Care Committee of the Fujian Medical University.

After 7 days of acclimation, a total of 144 mice received CT26 cells as subcutaneous injections in the right armpits, with a homogenate of $50 \mathrm{mg}\left(2-3 \mathrm{~mm}^{3}\right)$ minced solid murine CT26 adenocarcinoma in $0.1 \mathrm{ml}$ of saline, while 18 mice were injected with saline alone as healthy controls. The tumor-inoculated animals were randomly divided into the eight groups as follows: CAST, IV, calpeptin, CAST+IV, CAST+calpeptin, IV+calpeptin, CAST+IV+calpeptin, and cachexia control group $(\mathrm{n}=18$ per group). Intraperitoneal administration of CAST (1.5 $\mu \mathrm{g} / \mathrm{kg}$, daily), IV (0.75 mg/kg, daily), and/or calpeptin $(1.75 \mathrm{mg} / \mathrm{kg}$, daily) at a total volume of $0.2 \mathrm{ml}$ began on the 12th day after inoculation, when the mice presented signs and symptoms of cachexia, and lasted for 7 consecutive days. Besides the above mentioned healthy controls, 18 mice of the cachexia control group also received an equal volume of DMSO+saline i.p. once daily for one week in parallel. Physical activity, fur condition, body weight, food intake, and tumor growth were recorded daily. The tumor volume was calculated according to the following method: tumor volume $\mathrm{V}\left(\mathrm{cm}^{3}\right)=\left(\mathrm{axb}^{2}\right) / 2$, where $a$ is the length and $b$ is the width.

On the 19th day, ten mice in each group were randomly sacrificed, and their blood, tumors, and gastrocnemius muscles were collected for further analysis. After being obtained from orbital veins and clotting for $1 \mathrm{~h}$, blood samples were centrifuged at $1200 \mathrm{x} \mathrm{g}$ for $10 \mathrm{~min}$, the serum was collected and stored at $-20^{\circ} \mathrm{C}$. After the animals were euthanized by cervical dislocation, both their gastrocnemius muscles from the left leg and tumors were harvested and weighed. The gastrocnemius muscles were then snap-frozen in liquid nitrogen and stored at $-80^{\circ} \mathrm{C}$. Survival time for the remaining eight mice from each group was monitored and analyzed.

Bioanalytical assays. Serum glucose, triglyceride, total protein, and albumin used as biochemical markers of nutritional status were measured by an Olympus AU2700 automated biochemistry analyzer (Olympus, Tokyo, Japan).

Calpain activity assay. Calpain activity was measured by a calpain activity assay kit (Biovision, Mountain View, CA, USA). An appropriate amount of muscle tissue was ground into powder in liquid nitrogen. The fine powder was then lysed with extraction buffer in the kit and placed on ice for $20 \mathrm{~min}$. After centrifuging in a microcentrifuge at 10,000 x g for $5 \mathrm{~min}$, the supernatants were collected. After protein quantification using a Bradford protein assay kit (Wanleibio, Shenyang, China), $100 \mu \mathrm{g}$ of protein was incubated with calpain substrate Ac-LLY-AFC in a dark fluorescent microplate at $37^{\circ} \mathrm{C}$ for $1 \mathrm{~h}$. The samples were read in a SpectraMax M5 microplate reader (Molecular Devices, Sunnyvale, CA, USA) equipped with a $400 \mathrm{~nm}$ excitation filter and a $505 \mathrm{~nm}$ emission filter.

Western blot analysis. The muscle specimens were homogenized and lysed in RIPA buffer mixed with protease inhibitors $(150 \mathrm{mM} \mathrm{NaCl}, 50 \mathrm{mM}$ Tris $\mathrm{pH} 7.4,1 \%$ Triton $\mathrm{X}-100,1 \%$ sodium deoxycholate, $0.1 \%$ sodium dodecyl sulfate, sodium orthovanadate, sodium fluoride, ethylenediaminetetraacetic acid, and leupeptin). After centrifugation at $12,000 \mathrm{xg}$ for $5 \mathrm{~min}$ at $4^{\circ} \mathrm{C}$, the resulting proteins were quantified with a bicinchoninic acid protein assay kit (Beyotime, Shanghai, China). Proteins were separated by SDS-polyacrylamide gel electrophoresis according to their molecular weight using a mini-gel electrophoresis system (Bio-Rad, Hercules, CA, USA) and subsequently transferred onto a polyvinylidene difluoride membrane (Millipore, Billerica, MA, USA). The membrane was blocked with $5 \%$ bovine serum albumin at room temperature for $1 \mathrm{~h}$, then incubated with primary antibodies overnight at $4^{\circ} \mathrm{C}$, and further incubated with horseradish peroxidase-conjugated anti-rabbit or mouse $\mathrm{IgG}$ for $1 \mathrm{~h}$ at room temperature. The protein bands were illuminated with enhanced chemiluminescence reagents (Gen-View Scientific, Inc., Calimesa, CA, USA) and detected by the ChemiDoc XRS+ system (Bio-Rad). Finally, optical density values were normalized to that of the housekeeping protein glyceraldehyde3-phosphate dehydrogenase (GAPDH) and analyzed with ImageJ computer software (NIH, Bethesda, MD, USA). The average values were obtained from three repeated measures. 


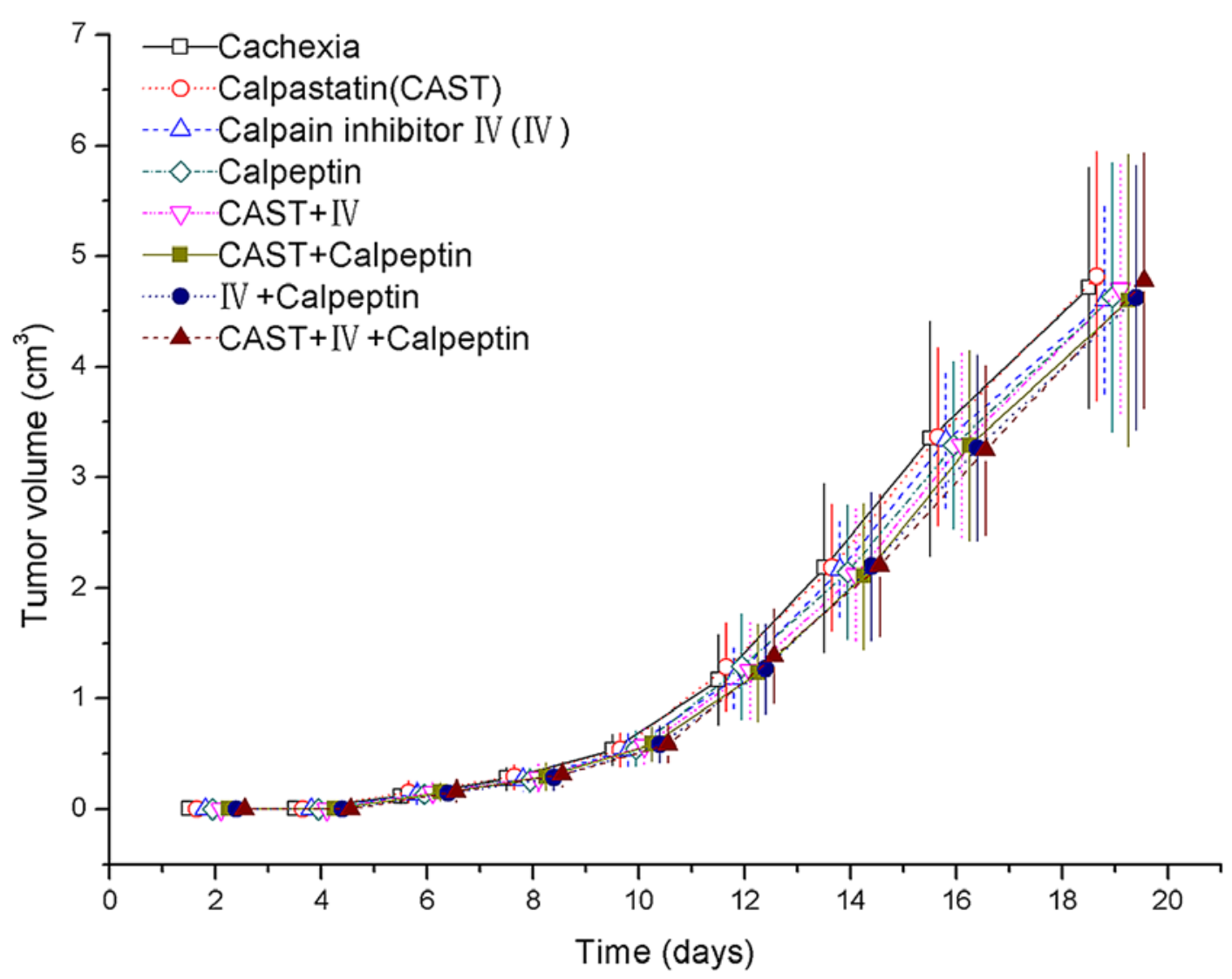

Figure 1. Effect of CAST, IV, and calpeptin on tumor volume $\left(\mathrm{cm}^{3}\right)$ of mice with cancer cachexia. Bars represent the mean $\pm \mathrm{SD}$, $\mathrm{n}=18$ in each group. From the day when tumors were perceptible to day 19 , there were no significant differences in tumor volume across all the groups or between any two groups ( $\mathrm{P}>0.05)$.

Quantitative real-time PCR. RNA of collected tissue was isolated using a tripure isolation reagent (Roche Diagnostics, Indianapolis, IN, USA). cDNA was synthesized from $3 \mu \mathrm{g}$ total RNA using the First Strand cDNA Synthesis kit (Gen-View Scientific, Inc.) according to the manufacturer's instructions. Semi-quantitative real-time PCR analysis was performed using an Applied Biosystems 7500 Real-time PCR System (Applied Biosystems, Carlsbad, CA, USA) with SYBRGreen staining of DNA double-strands, while the staining was performed using the FastStart Universal SYBR Green Master (Roche Diagnostics) according to the product manual. Primer pairs for the amplification of E3 ubiquitin ligase genes were the following: mouse MuRF-1 (sense: 5-GGAACACGAAGA CGAGAAAATC-3, antisense: 5-TGGCTATTCTCCTTGG TCACTC-3); mouse atrogin-1 (sense: 5-GAAGAGAGCAGT ATGGGGTCAC-3, antisense: 5-CTTGAGGGGAAAGRG AGACG-3); and mouse GAPDH (sense: 5-GGTGAAGGTCG GTGTGAACG-3, anti-sense: 5-CTCGCTCCTGGAAGAT GGTG-3). The amplification conditions were as follows: $50^{\circ} \mathrm{C}$ for $2 \mathrm{~min}, 95^{\circ} \mathrm{C}$ for $10 \mathrm{~min}$, and 40 cycles at $95^{\circ} \mathrm{C}$ for $15 \mathrm{sec}$ and $60^{\circ} \mathrm{C}$ for $1 \mathrm{~min}$. Finally, either target gene expression was normalized to the housekeeping gene mouse GAPDH that was amplified at the same time, and further analyzed using the $2^{-\Delta \Delta C T}$ method (14).

Statistical analyses. All data were expressed as mean \pm standard deviation. Differences were examined for significance using one-way ANOVA followed by Tukey's pos-hoc test with SPSS 19.0 software (SPSS, Chicago, IL, USA). The
Kruskal-Wallis test followed by the Nemenyi test was used when the data distribution was skewed. The log-rank test was used for survival analysis. All statistical analyses were two-sided, and P-values of 0.05 were considered statistically significant.

\section{Results}

Tumor volume. The tumors of CT26-inoculated mice were perceptible by day 6 , and grew more rapidly from day 10 onward. The mice exhibited symptoms of cachexia on day 12 after tumor inoculation, including delayed responsiveness, poor physical activity, scruffy fur, piloerection, and darkening of dorsal hairs. All the tumor-bearing groups had a significant increase in tumor volume over time but no significant differences were observed between the treatment groups and the cachexia control group (Fig. 1).

Tumor-free body weight. There was no difference in initial body weight among the nine groups (Fig. 2). As the body weight of the healthy control group grew during the study period, the weight of the tumor-bearing groups increased during the first 8 days then declined from day 9 to the final day, despite a slight transient increase in some groups (Fig. 2). From days 16-19, the tumor-free body weight of the IV+calpeptin and CAST+IV+calpeptin groups were significantly higher than the cachexia group $(\mathrm{P}=0.03, \mathrm{P}=0.016$ and $\mathrm{P}=0.015, \mathrm{P}=0.011$, respectively). A significant tumor-free body weight increase was observed in the calpeptin, CAST+IV, and CAST+calpeptin groups only on day $19(\mathrm{P}=0.045, \mathrm{P}=0.045$, 


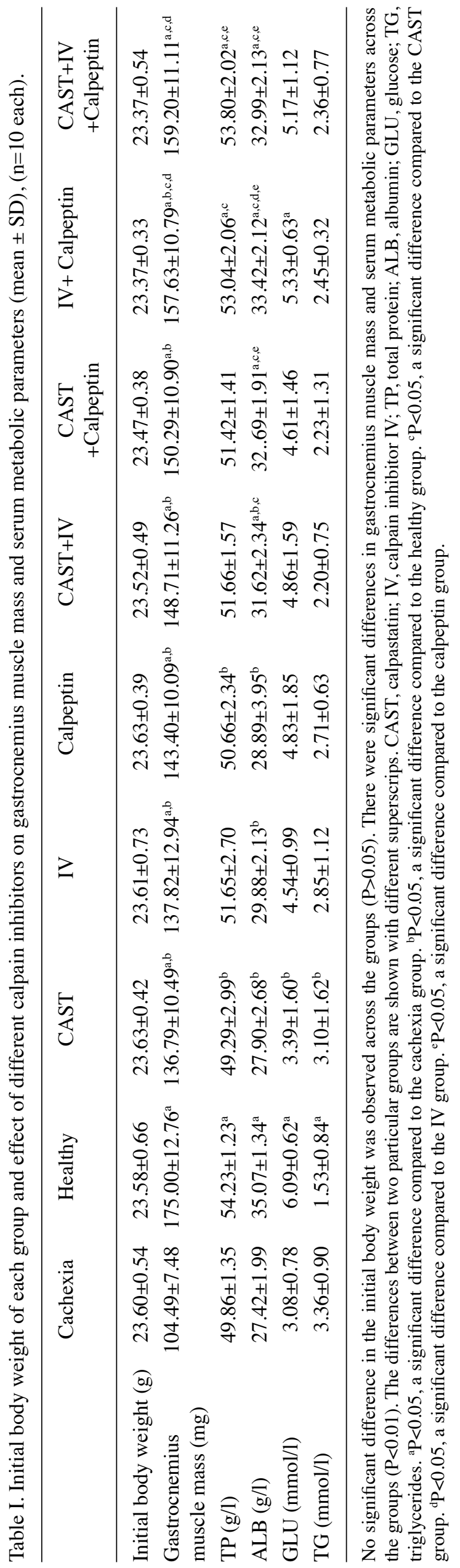

$\mathrm{P}=0.014$, respectively). However, the tumor-free body weight of all tumor-bearing groups was significantly different from that of the healthy controls from days $12-19(\mathrm{P}<0.01)$. Cumulative food intake of standard chow showed no significant difference during the experiment (data not shown).

Muscle weight and serum nutritional markers. Significant loss of gastrocnemius wet weight was found in the cachexia controls as compared to the healthy controls (Table I, $\mathrm{P}<0.001$ ). There were also significant differences in gastrocnemius weight between any treatment group and the cachexia group $(\mathrm{P}<0.001)$. Moreover, a markedly increased gastrocnemius muscle mass was observed in the IV+Calpeptin and CAST+IV+calpeptin groups compared with the CAST and IV groups $(\mathrm{P}=0.002$, $\mathrm{P}=0.001$ and $\mathrm{P}=0.004, \mathrm{P}=0.001$, respectively).

To evaluate the effect of treatments with different calpain inhibitors on the nutritional state of cachectic mice, we measured the serum levels of serum total protein, albumin, glucose, and triglycerides in the nine groups. Mice from the cachexia group had lower levels of total protein, albumin, and glucose, and higher levels of triglycerides (Table I, $\mathrm{P}<0.01$ ) compared with the healthy controls. The administration of different calpain inhibitors could partially reverse these metabolic changes, especially the combination treatment with two or three kinds of inhibitors. However, no significant difference in these markers was found between any mono-treatment group and the cachexia controls (between the CAST group and the cachexia group, $\mathrm{P}=0.999, \mathrm{P}=1.000$, $\mathrm{P}=1.000, \mathrm{P}=1.000$; between the IV group and the cachexia group, $\mathrm{P}=0.581, \mathrm{P}=0.352, \mathrm{P}=1.000, \mathrm{P}=0.961$; between the calpeptin group and the cachexia group, $\mathrm{P}=0.994, \mathrm{P}=0.903$, $\mathrm{P}=0.392, \mathrm{P}=0.862$ ).

Survival time. To evaluate the effect of the different treatments on the survival time of mice, we constructed Kaplan-Meier curves with 8 mice in each group (eight remained alive after the other ten were sacrificed on day 19). The data demonstrated that most of the treatment groups had a longer survival time than the cachexia group (Fig. 3, P<0.05) except for the calpastatin group $(\mathrm{P}=0.101)$. In addition, there were no significant differences among the different treatment groups $(\mathrm{P}>0.05)$. All mice in the healthy control group survived for 50 days.

Calpain expression and activity. Previous studies (15) have shown that activation of the calpain system is ubiquitous in cancer cachexia, and calpain inhibitors can antagonize the effects induced by activated calpain. To confirm that treatments with different calpain inhibitors affect calpain expression and activity in cachectic mice, we measured the levels of $\mu$-calpain, $\mathrm{m}$-calpain, and calpastatin, and examined the calpain activity in all nine groups.

There were no significant differences in either $\mu$-calpain or m-calpain protein expression between the cachexia controls and the healthy controls. Treatment with calpain inhibitor IV and calpeptin were associated with lower levels of the two ubiquitous calpains; however, their levels in the calpastatin group were higher than the control groups and the other monotreatment groups, while their expression could be downgraded when combined with other calpain inhibitors such as calpain inhibitor IV, calpeptin, or both (Fig. 4A, B and D). 


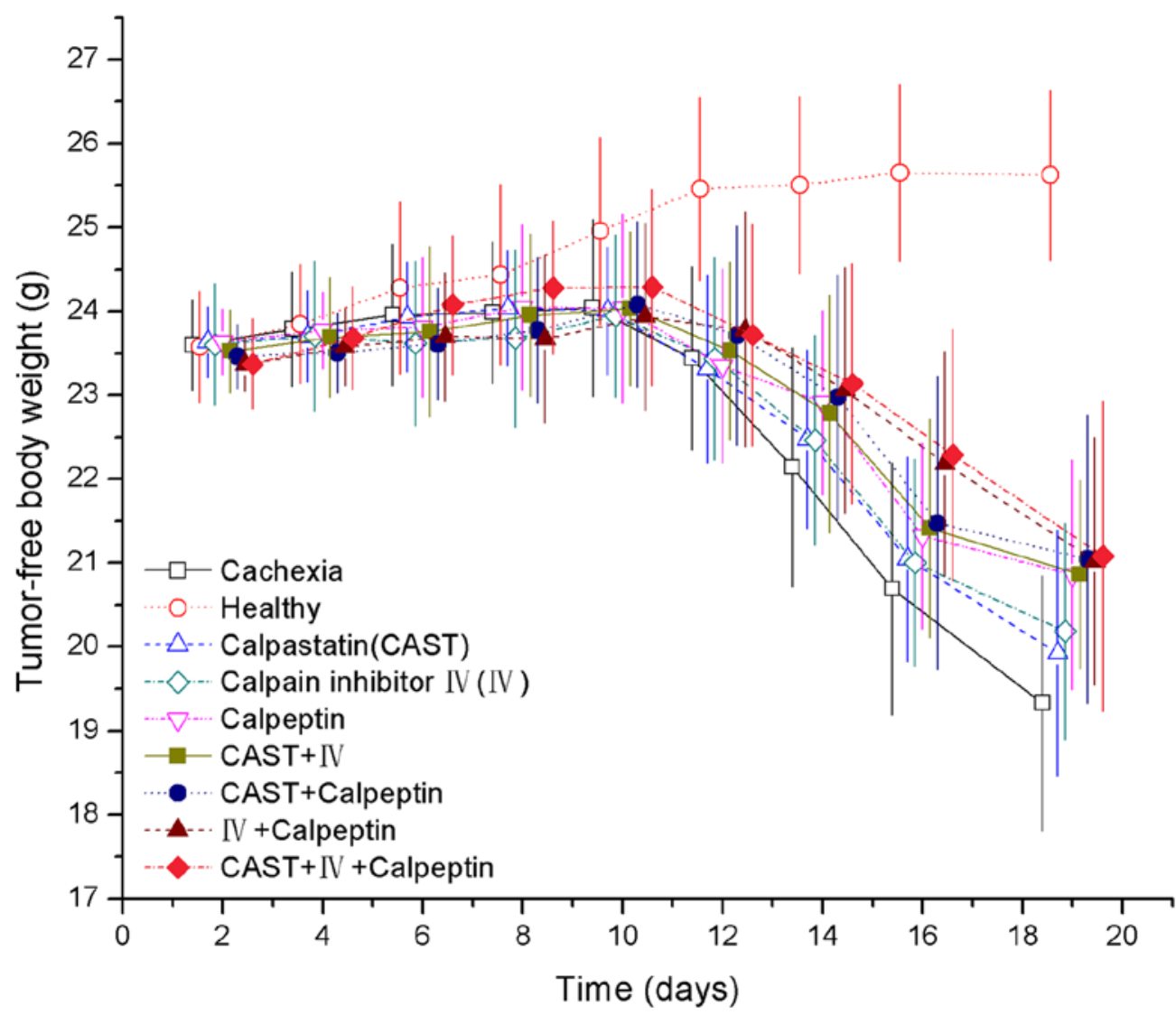

Figure 2. Effect of CAST, IV, and calpeptin on tumor-free body weight ( $\mathrm{g}$ ) of mice with cancer cachexia. Bars represent mean $\pm \mathrm{SD}, \mathrm{n}=18$ in each group. There were significant differences across all the groups on days 12, 14, 16 and $19(\mathrm{P}<0.01)$. Pair-wise comparisons showed that (A) each of the eight tumor-bearing groups was significantly different from the healthy group on days 12, 14, 16 and $19(\mathrm{P}<0.01)$. (B) The calpeptin group (day 19), CAST+IV group (day 19), CAST+calpeptin group (day 19), IV+calpeptin group (days 16 and 19) and CAST+IV+calpeptin group (days 16 and 19) were significantly different from the cachexia group $(\mathrm{P}<0.05)$; and $(\mathrm{C})$ for each day, there were no significant differences across the treatment groups or between any two particular groups $(\mathrm{P}>0.05)$.

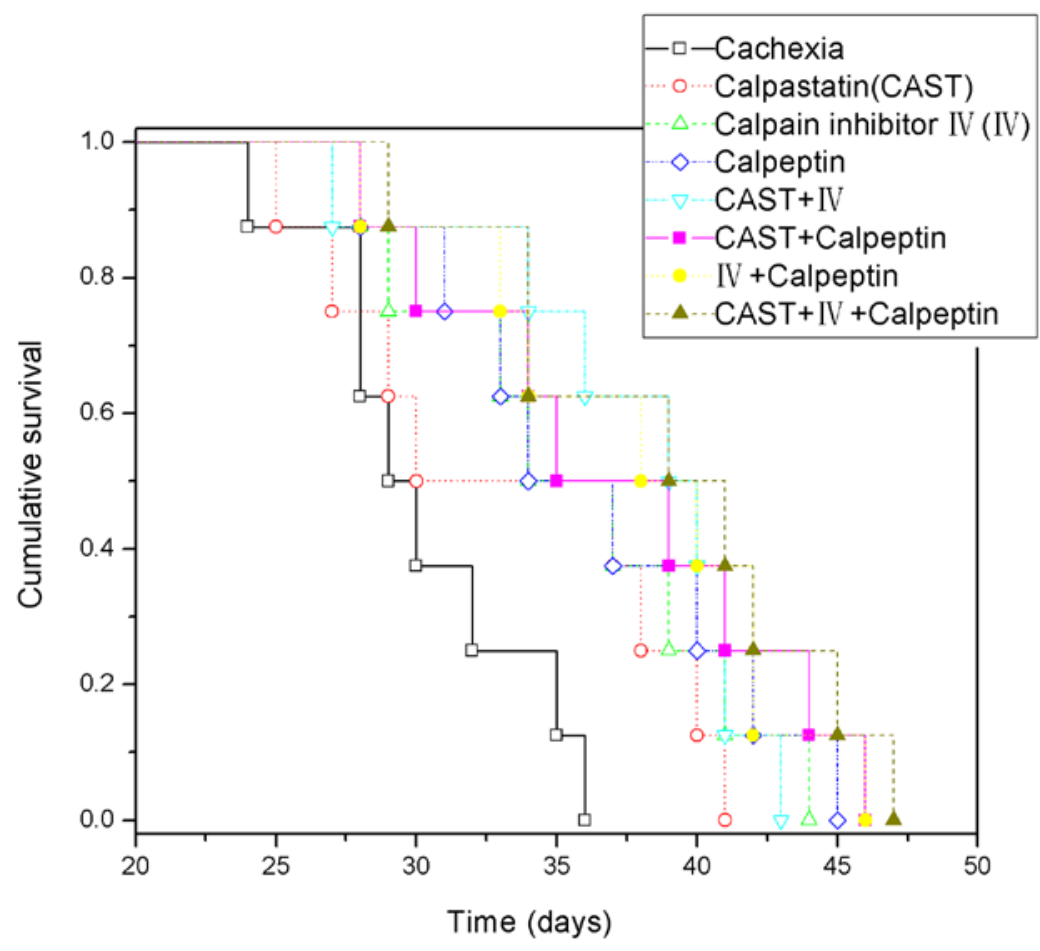

Figure 3. Kaplan-Meier survival curves of mice in each of the eight tumor-bearing groups ( $\mathrm{n}=8$ each). The curves were significantly different across all treatment groups (log-rank test, $\mathrm{P}=0.007)$. All treatment groups had a markedly longer survival time than the cachexia group $(\mathrm{P}<0.05)$, except for the calpastatin group $(\mathrm{P}=0.101)$. 

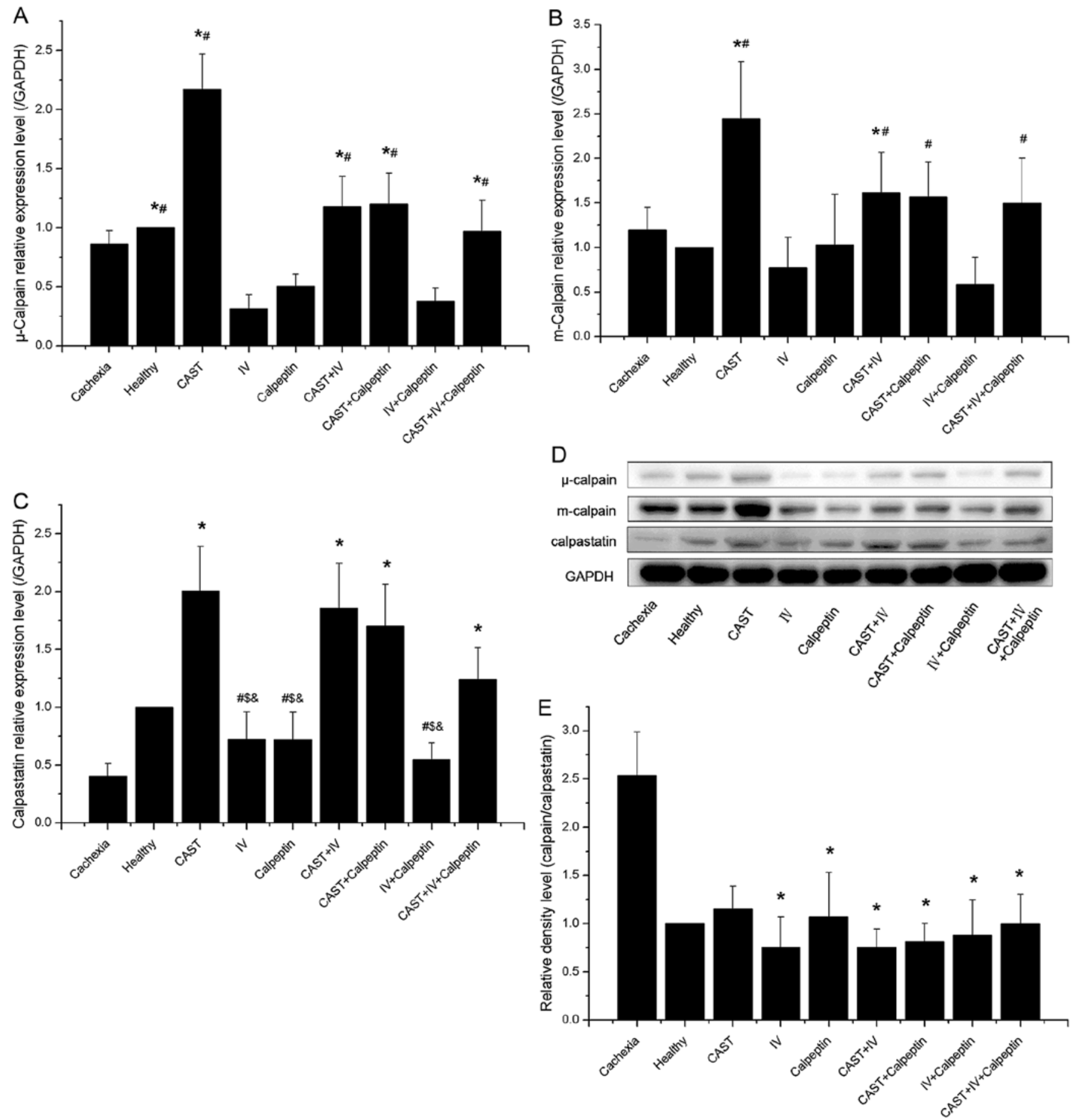

Figure 4. Effect of different inhibitor treatments on the calpain system in tumor-bearing mice administered after CT26 inoculation and control groups (n=10 each). (A) The level of $\mu$-calpain in gastrocnemius muscle extract. Significant differences were detected between the IV and the healthy groups ("P=0.005), between the IV group and the groups involving calpastatin ( ${ }^{*} \mathrm{P}<0.001 ; \mathrm{P}=0.011$ when IV versus CAST+IV+calpeptin), between the IV+calpeptin and the healthy groups $\left({ }^{*} \mathrm{P}=0.021\right)$, and between the IV+calpeptin group and the groups involving calpastatin $\left({ }^{*} \mathrm{P}<0.001, \mathrm{P}=0.001, \mathrm{P}=0.001, \mathrm{P}=0.045\right.$, respectively). (B) The level of m-calpain in gastrocnemius muscle extract. Significant differences were observed between the IV and the CAST groups ("P<0.001), between the IV and the CAST+IV groups ( $\mathrm{P}=0.023)$, and between the IV+calpeptin group and the groups involving calpastatin $\left({ }^{*} \mathrm{P}<0.001, \mathrm{P}=0.001, \mathrm{P}=0.002, \mathrm{P}=0.015\right.$, respectively). (C) The level of calpastatin in gastrocnemius muscle extract. Significant differences were observed between the cachexia group and the groups involving calpastatin ( $\mathrm{P}<0.001 ; \mathrm{P}=0.005$ when cachexia versus $\mathrm{CAST}+\mathrm{IV}+$ calpeptin), between the CAST group and the treatment groups not involving calpastatin $\left({ }^{*} \mathrm{P}=0.001, \mathrm{P}=0.001, \mathrm{P}<0.001\right.$, respectively), between the CAST $+\mathrm{IV}$ group and the treatment groups not involving calpastatin ( ${ }^{\$} \mathrm{P}=0.003, \mathrm{P}=0.002$, $\mathrm{P}<0.001$, respectively), and between the CAST+calpeptin group and the treatment groups not involving calpastatin ( ${ }^{\circledR} \mathrm{P}=0.009, \mathrm{P}=0.008, \mathrm{P}<0.001$, respectively). (D) The protein bands of $\mu$-calpain, m-calpain, calpastatin, and the housekeeping protein GAPDH. (E) The calpain-to-calpastatin ratio. Significant differences were observed between the cachexia group and the treatment groups ( $\mathrm{P}<0.001, \mathrm{P}=0.018, \mathrm{P}<0.001, \mathrm{P}<0.001, \mathrm{P}=0.001, \mathrm{P}=0.028$, respectively) except for the CAST group $(\mathrm{P}=0.697)$.

Lower levels of calpastatin were observed in the muscle of tumor-bearing mice compared to the healthy controls, except for the groups affected by exogenous calpastatin. Compared with groups not involving calpastatin, there were 


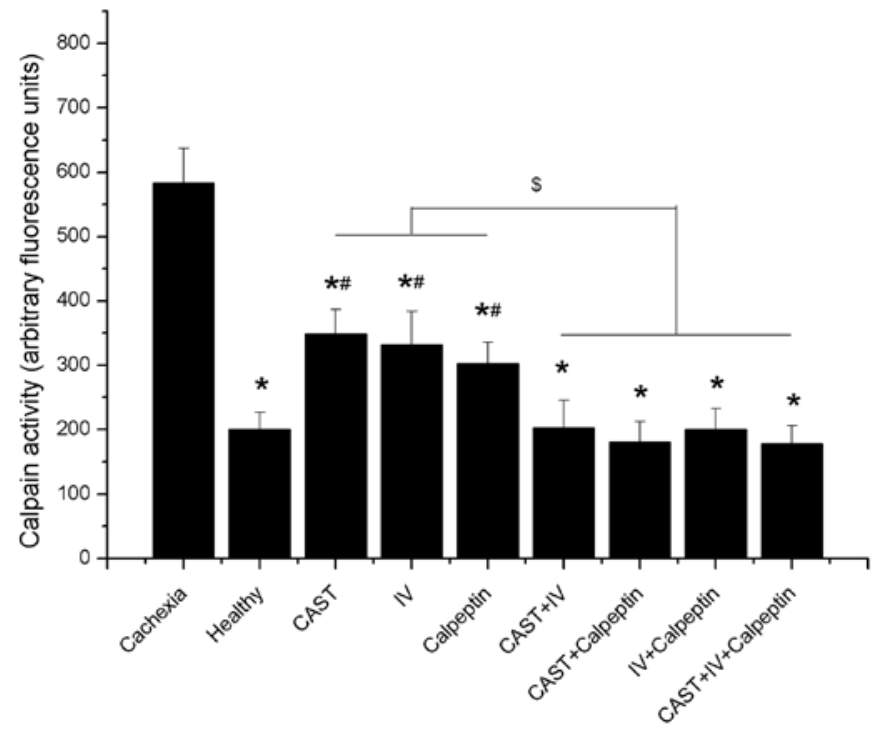

Figure 5. Calpain activity in the gastrocnemius muscle extract of each group $(\mathrm{n}=10$ each). A significant difference between the cachexia group and any other group ( $\mathrm{P}<0.001)$. A significant difference between the healthy group and any mono-treatment group $\left({ }^{*} \mathrm{P}<0.001\right)$. A significant difference between any mono-treatment group and any combined treatment group $\left({ }^{\$} \mathrm{P}<0.001\right)$.

significant higher levels of calpastatin in the groups treated with calpastatin peptide alone or combined with other inhibitors. In addition, administration with other inhibitors could decrease the levels of calpastatin (Fig. 4C and D).

In accordance with a previous study (16), we found that both the levels of $\mu$-calpain and m-calpain remained unchanged in the cachexia and healthy controls during the experiment $(\mathrm{P}>0.05)$, but the calpastatin level showed a progressive decrease after tumor transplantation, resulting in a rising imbalance of the calpain/calpastatin ratio. Moreover, the ratio of calpain to calpastatin had a positive correlation with calpain activity (Figs. 4E and 5). The present study indicated that the levels of $\mu$-calpain, $\mathrm{m}$-calpain, and calpastatin expression in tumor-bearing mice were affected by different inhibitors and that the ratio of calpain to calpastatin and the calpain activity was reduced through the administration of any inhibitor alone or in combination.

Expression of atrogin-1 and MuRF-1 mRNA. To characterize the effects of calpain inhibitors on muscle atrophy, mRNA levels of atrogin-1 (MAFbx) and MuRF-1 were measured. Results from qRT-PCR analyses revealed that atrogin-1 and MuRF-1 were significantly greater in all cachexia mice than in the healthy controls (approximately a 20 -fold increase). The mono-treatment of calpain inhibitors significantly downgraded MuRF-1 levels by 74,79 , and $56 \%$ and atrogin- 1 levels by 58 , 75 , and $61 \%$, respectively, compared to the cachexia control group. Further reductions were observed when these inhibitors were administrated together (Fig. 6A and B).

Expression of apoptosis-related protein. To evaluate the effect of calpain inhibitors on apoptosis, we measured the expression of several widely used apoptosis-related proteins, including caspase-3, BAX, and BCL-2. Our results confirmed that apoptosis was present in skeletal muscle of cachectic mice, as there
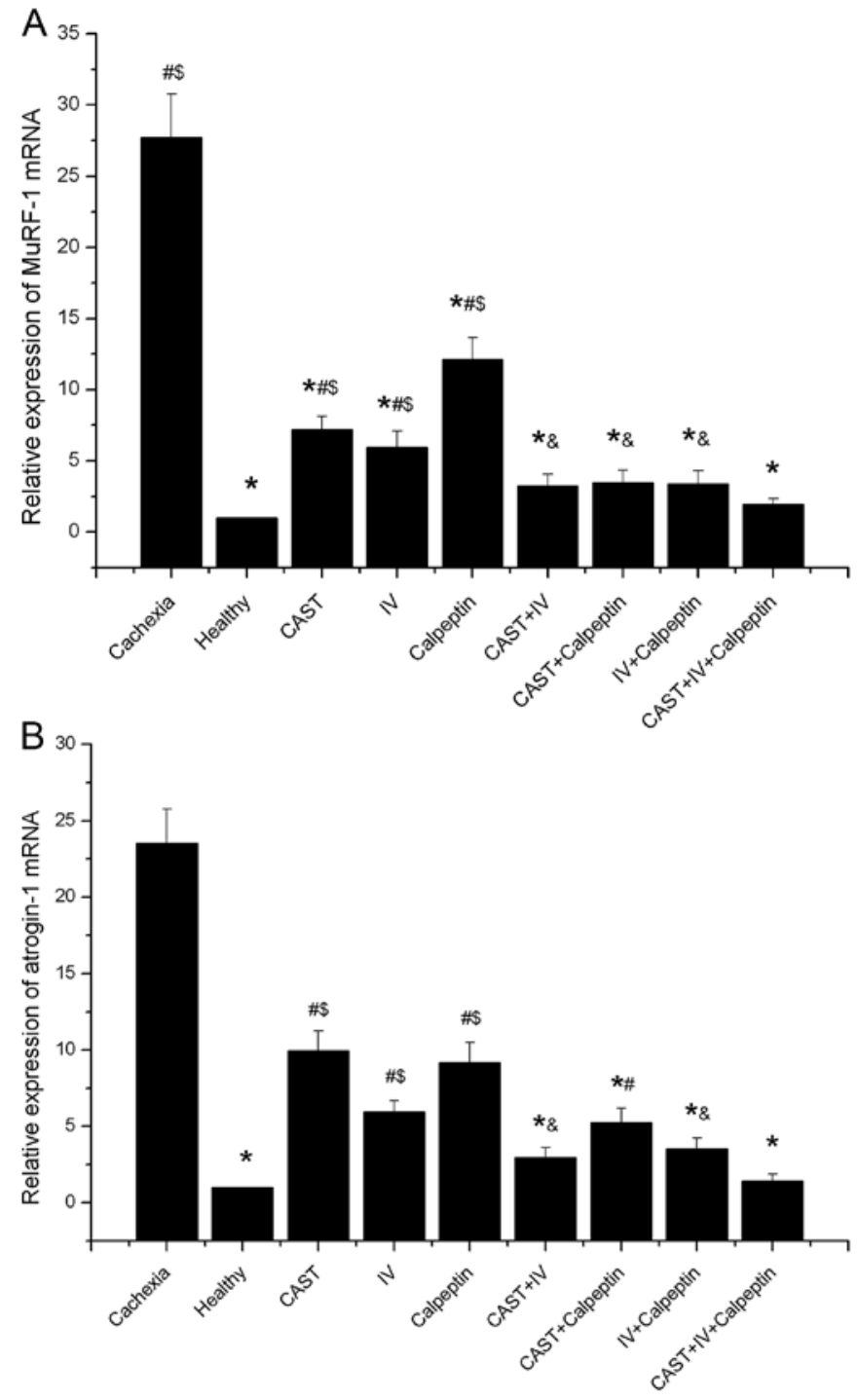

Figure 6. Effect of different inhibitor treatments on MuRF-1 and atrogin-1 mRNA expression ( $\mathrm{n}=10$ each). (A) The relative expression of MuRF-1 mRNA. A significant difference was observed between the cachexia group and the healthy group, between the cachexia group and any combined treatment group $\left({ }^{*} \mathrm{P}<0.01\right)$, between the healthy group and any mono-treatment group $\left({ }^{*} \mathrm{P}<0.001\right)$, and between the CAST $+\mathrm{IV}+$ calpeptin group and any mono-treatment group $\left({ }^{\mathrm{S}} \mathrm{P}<0.05 ; \mathrm{P}=0.003, \mathrm{P}=0.025, \mathrm{P}<0.001\right.$, respectively). In addition, a significant difference was also found between the calpeptin group and any combined treatment group $\left({ }^{\circledR} \mathrm{P}<0.05 ; \mathrm{P}=0.012, \mathrm{P}=0.029\right.$ $\mathrm{P}=0.02$, respectively). (B) The relative expression of atrogin-1 mRNA. A significant difference was found between the cachexia group and the healthy group, between the cachexia group and any combined treatment group $\left({ }^{*} \mathrm{P}<0.01\right)$, between the healthy group and any mono-treatment group $\left({ }^{*} \mathrm{P}<0.01\right.$; $\mathrm{P}<0.001, \mathrm{P}=0.004, \mathrm{P}<0.001$, respectively), between the healthy group and the CAST+calpeptin group $\left({ }^{*} \mathrm{P}=0.021\right)$, and between the CAST+IV+calpeptin group and any mono-treatment group $\left({ }^{\$} \mathrm{P}<0.05 ; \mathrm{P}<0.001, \mathrm{P}=0.028, \mathrm{P}<0.001\right.$, respectively). In addition, a significant difference was also observed between the CAST group and the CAST+IV group $\left({ }^{\&} \mathrm{P}=0.007\right)$, between the CAST group and the IV+calpeptin group $\left({ }^{\&} \mathrm{P}=0.032\right)$.

were significantly higher caspase-3 and BAX protein levels and lower BCL-2 levels in cachexia control animals as compared to those observed in the healthy animals. Mono-treatment with calpain inhibitor IV, calpeptin, or both was associated with a decrease in the expression of caspase- 3 and BAX and an increase in the expression of BCL-2, which decreased the BAX-to-BCL-2 ratio. Conversely, administration of calpastatin 

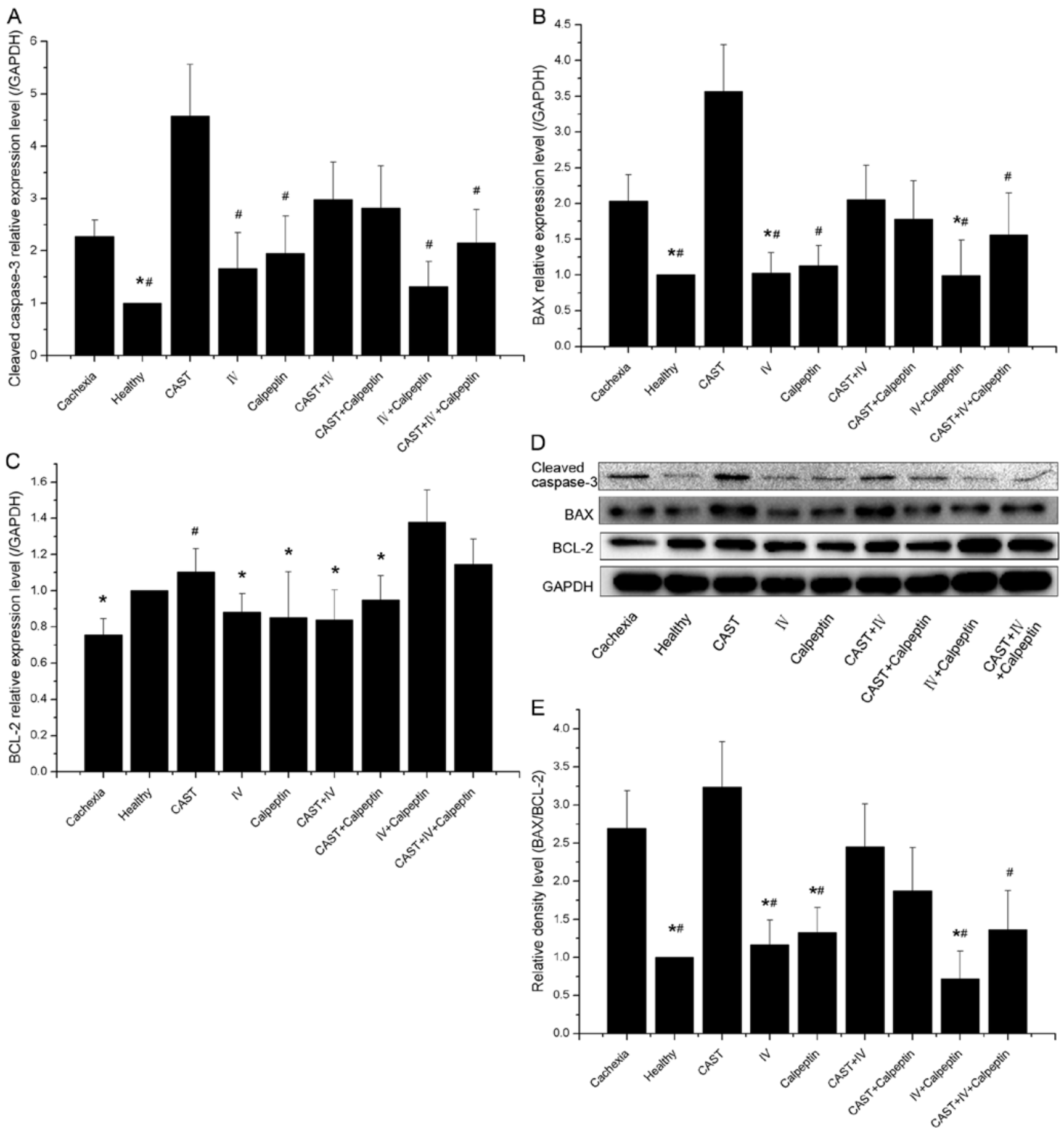

Figure 7. Effect of different inhibitor treatments on muscle apoptosis in tumor-bearing and control mice ( $\mathrm{n}=10$ each). (A) The level of cleaved caspase-3 in gastrocnemius muscle extract. Significant differences were detected between the cachexia group and the healthy group (" $\mathrm{P}=0.017)$, between the CAST group and any other group $\left({ }^{\sharp} \mathrm{P}<0.001, \mathrm{P}<0.001, \mathrm{P}=0.011, \mathrm{P}<0.001, \mathrm{P}=0.033\right.$, respectively) except when compared with the CAST $+\mathrm{IV}$ and the CAST + calpeptin groups $(\mathrm{P}>0.05)$. (B) The level of BAX in gastrocnemius muscle extract. Significant differences were found between the cachexia group and the healthy group ( $\mathrm{P}=0.005)$, between the cachexia group and the IV group ( $\left.{ }^{*} \mathrm{P}=0.021\right)$, between the cachexia group and the IV+calpeptin group ( $\left.\mathrm{P}=0.021\right)$, and between the CAST group and any other group $\left({ }^{\prime \prime} \mathrm{P}<0.001 ; \mathrm{P}=0.035\right.$ when CAST versus CAST+IV+calpeptin) except when compared with the cachexia, the CAST+IV and the CAST+calpeptin groups $(\mathrm{P}>0.05)$. (C) The level of BCL-2 in gastrocnemius muscle extract. Significant differences were observed between the IV+calpeptin group and any other group ( $\mathrm{P}<0.001 ; \mathrm{P}=0.024$ when IV+calpeptin versus CAST+calpeptin) except when compared with the healthy, the CAST and the CAST+IV+calpeptin groups $(\mathrm{P}>0.05)$. In addition, a significant difference was also found between the cachexia group and the $\mathrm{CAST}$ group $\left({ }^{\#} \mathrm{P}=0.002\right)$. (D) The bands of cleaved caspase-3, BAX, BCL-2, and the housekeeping protein GAPDH. (E) The BAX-to-BCL-2 ratio. Significant differences were observed between the healthy group and the cachexia group ("P $<0.001$ ), between the IV+calpeptin group and the cachexia group ("P<0.001), between the IV group and the cachexia group $\left({ }^{*} \mathrm{P}=0.008\right)$, between the calpeptin group and the cachexia group $\left({ }^{*} \mathrm{P}=0.047\right)$, and between the CAST group and any other group $\left({ }^{\#} \mathrm{P}<0.001\right.$, $\mathrm{P}<0.001, \mathrm{P}=0.007, \mathrm{P}<0.001, \mathrm{P}=0.011$, respectively) except when compared with the cachexia, the CAST+calpeptin and the CAST $+\mathrm{IV}$ groups $(\mathrm{P}>0.05)$.

peptide increased the protein levels of caspase- 3 and BAX, and reduced the expression of BCL-2, although combination with other calpain inhibitors, such as calpain inhibitor IV, calpeptin, or both, could alleviate these changes to some extent (Fig. 7A-E). 


\section{Discussion}

Cancer cachexia, a debilitating and life-threatening syndrome, occurs in $22-55 \%$ of patients with advanced colorectal cancer and is associated with high morbidity and mortality rates (17). As no effective treatment currently exists, only palliative care is available for patients (18). Given that skeletal muscle loss is the primary symptom of cancer cachexia, and that the calpain system and the ubiquitin-proteasome pathway (UPP) are critical to proteolysis, we hypothesized that calpain might act upstream of UPP (11) and that calpain inhibition could further reduce UPP-dependent protein breakdown.

After administration with calpain inhibitors, we observed significant increase in tumor-free body weight and gastrocnemius muscle mass among the treatment groups compared with the cachexia control group. In addition, combining multiple agents was more effective at preserving body weight than mono-treatments. Most of the treatment groups had a longer survival time than the cachexia group, indicating that the treated tumor-bearers might benefit from calpain inhibition. In addition, no statistical difference in tumor mass was observed among the groups of tumor-bearing mice, suggesting that these agents have no adverse effects on tumor growth in the current study. These results support the concept that calpain inhibitor treatment can alleviate the muscle loss induced by cachexia.

In this study, the calpain system was activated in the skeletal muscles of cachectic mice, resulting from an increasing calpain/calpastatin ratio instead of increased calpains in absolute amounts, as also seen in previous studies (19). Calpain inhibitor IV and calpeptin (pharmacological calpain inhibitors) could suppress calpain activity by reducing their amounts, but calpastatin (an endogenous calpain inhibitor) downregulated the activity of the calpain system by raising the level of calpastatin and further decreasing the calpain/ calpastatin ratio. The fact that the calpastatin treatment group had a significantly higher level of calpain than the other groups might indicate a positive feedback mechanism. Interestingly, we found pharmacological calpain inhibitors could ameliorate the excessive increase of calpains caused by calpastatin during combination treatment, suggesting that combination therapy might be an effective strategy for reversing the abnormal activation of the calpain system.

The unique ubiquitin E3 ligases, muscle atrophy F-box (MAFbx or atrogin-1), and muscle ring finger-1 (MuRF-1), essential for the ubiquitin-proteasome pathway, are upregulated in the process of skeletal muscle atrophy (20). Atrogin-1 or MuRF1 gene knockouts were confirmed to be resistant to muscle atrophy induced by different conditions $(21,22)$. Previous studies indicated that activation of UPP might be the consequence of a growing number of substrates produced by the activated calpain system rather than the causative factors of muscle wasting (19). Our study provides further support for this concept: we found that there was a positive correlation between atrogin-1/MuRF1 mRNA expression and calpain activity when they were downregulated synchronously. Furthermore, our data suggest that enhancing the inhibition action of calpains through combination therapy could lower the level of atrogin-1/MuRF1 mRNA, protecting against muscle atrophy. However, further studies will clarify how cell signaling pathways take part in the linkage between UPP and the calpain system.

During the past several decades, increasing numbers of reports have supported the concept that apoptosis is a common feature in skeletal muscle of tumor-bearing animals and patients with cancer-related cachexia $(9,23)$. Apoptosis may account for muscle wasting under cachectic condition as well as the abnormally activated proteolytic system. In the present study, the observation that all tumor-bearing mice had significantly higher levels of cleaved caspase-3 and BAX and lower levels of BCL-2 compared to healthy controls, is consistent with previous studies (24). In addition, the administration of calpain inhibitor IV and calpeptin alleviated skeletal muscle apoptosis was partly through reducing the activation of caspase- 3 and decreasing the BAX-to-BCL2 ratio, as other calpain inhibitors have been shown to do during the process of injury-induced neuronal apoptosis $(25,26)$. However, the calpastatin treatment group exhibited more apoptotic proteins (cleaved caspase-3 and BAX) than other groups, which might be attributed to its higher levels of calpains and calpastatin (27) and which could be ameliorated during combination treatment with pharmacological calpain inhibitors.

In our study, calpain inhibitor IV and calpeptin alone and in combination could partially reverse tumor-free body weight loss and muscle wasting, while calpastatin alone was less effective due to increased calpain levels and apoptosis. Furthermore, our doses of these inhibitors were derived from previous reports and were not optimized by titration, which might lead to an insufficient inhibition of calpain and nonsignificant differences between groups. Although no adverse effects on tumor growth or shortened survival were observed in our study, other side effects might occur in vivo. Nevertheless, future studies should focus on the pharmacokinetics and toxicology of these agents when administrated together, as well as their underlying mechanisms of action, before they enter into the next phase of clinical research.

In conclusion, our study showed that treatment of cachectic tumor-bearing mice with three different calpain inhibitors, alleviated cachexia-associated symptoms, increased tumorfree body weight and muscle mass. In addition, improved concentrations of serum nutritional markers and apoptosisrelated proteins in skeletal muscle, reduced calpain activity and E3 ubiquitin ligase expression were observed. Although the exact mechanism of calpain inhibitor in cachexia has not been elucidated fully and needs to be studied further to prove their security, our results may lead to the development of novel strategies for the prevention and treatment of cancer cachexia.

\section{Acknowledgements}

This work was supported by the National Natural Science Foundation of China (81272465).

\section{References}

1. Fearon K, Strasser F, Anker SD, Bosaeus I, Bruera E, Fainsinger RL, Jatoi A, Loprinzi C, MacDonald N, Mantovani G, et al: Definition and classification of cancer cachexia: An international consensus. Lancet Oncol 12: 489-495, 2011.

2. Baracos VE: Pitfalls in defining and quantifying cachexia. J Cachexia Sarcopenia Muscle 2: 71-73, 2011. 
3. von Haehling S and Anker SD: Cachexia as major underestimated unmet medical need: Facts and numbers. Int $\mathrm{J}$ Cardiol 161: 121-123, 2012.

4. Tisdale MJ: Molecular pathways leading to cancer cachexia. Physiology (Bethesda) 20: 340-348, 2005.

5. Del Fabbro E: Current and future care of patients with the cancer anorexia-cachexia syndrome. Proc ASCO: e229-237, 2015. 10.14694/EdBook_AM.2015.35.e229.

6. Goll DE, Neti G, Mares SW and Thompson VF: Myofibrillar protein turnover: The proteasome and the calpains. J Anim Sci 86 (Suppl): E19-E35, 2008.

7. Wing SS, Lecker SH and Jagoe RT: Proteolysis in illness-associated skeletal muscle atrophy: From pathways to networks. Crit Rev Clin Lab Sci 48: 49-70, 2011.

8. Johns N, Stephens NA and Fearon KC: Muscle wasting in cancer. Int J Biochem Cell Biol 45: 2215-2229, 2013.

9. Busquets S, Deans C, Figueras M, Moore-Carrasco R, LópezSoriano FJ, Fearon KC and Argilés JM: Apoptosis is present in skeletal muscle of cachectic gastro-intestinal cancer patients Clin Nutr 26: 614-618, 2007.

10. Donkor IO: Calpain inhibitors: a survey of compounds reported in the patent and scientific literature. Expert Opinion on Therapeutic Patents 21: 601-636, 2011. doi: 10.1517/13543776.2011.568480.

11. Huang $\mathrm{J}$ and Zhu X: The molecular mechanisms of calpains on muscle atrophy. Physiol Res 65: 547-560, 2016.

12. Zhang Z, Huang Z, Dai H, Wei L, Sun S and Gao F: Therapeutic efficacy of E-64-d, a selective calpain inhibitor, in experimental acute spinal cord injury. Biomed Res Int 2015: 134242, 2015.

13. Wang C, Shi D, Song X, Chen Y, Wang L and Zhang X: Calpain inhibitor attenuates ER stress-induced apoptosis in injured spinal cord after bone mesenchymal stem cells transplantation. Neurochem Int 97: 15-25, 2016.

14. Livak KJ and Schmittgen TD: Analysis of relative gene expression data using real-time quantitative PCR and the 2(-Delta Delta C(T)) method. Methods 25: 402-408, 2001.

15. Donkor IO: An updated patent review of calpain inhibitors (2012-2014). Expert Opin Ther Pat 25: 17-31, 2015.

16. Salamino F, De Tullio R, Mengotti P, Viotti PL, Melloni E and Pontremoli S: Different susceptibility of red cell membrane proteins to calpain degradation. Arch Biochem Biophys 298: 287-292, 1992.

17. Thoresen L, Frykholm G, Lydersen S, Ulveland H, Baracos V, Prado CM, Birdsell L and Falkmer U: Nutritional status, cachexia and survival in patients with advanced colorectal carcinoma. Different assessment criteria for nutritional status provide unequal results. Clin Nutr 32: 65-72, 2013.
18. Amano K, Maeda I, Morita T, Okajima Y, Hama T, Aoyama M, Kizawa Y, Tsuneto S, Shima Y and Miyashita M: Eating-related distress and need for nutritional support of families of advanced cancer patients: A nationwide survey of bereaved family members. J Cachexia Sarcopenia Muscle 7: 527-534, 2016.

19. Costelli P, De Tullio R, Baccino FM and Melloni E: Activation of $\mathrm{Ca}(2+)$-dependent proteolysis in skeletal muscle and heart in cancer cachexia. Br J Cancer 84: 946-950, 2001.

20. Foletta VC, White LJ, Larsen AE, Léger B and Russell AP: The role and regulation of MAFbx/atrogin-1 and MuRF1 in skeletal muscle atrophy. Pflugers Arch 461: 325-335, 2011.

21. Bodine SC, Latres E, Baumhueter S, Lai VK, Nunez L, Clarke BA, Poueymirou WT, Panaro FJ, Na E, Dharmarajan K, et al: Identification of ubiquitin ligases required for skeletal muscle atrophy. Science 294: 1704-1708, 2001.

22. Furlow JD, Watson ML, Waddell DS, Neff ES, Baehr LM, Ross AP and Bodine SC: Altered gene expression patterns in muscle ring finger 1 null mice during denervation- and dexamethasone-induced muscle atrophy. Physiol Genomics 45: 1168-1185, 2013.

23. van Royen M, Carbó N, Busquets S, Alvarez B, Quinn LS, López-Soriano FJ and Argilés JM: DNA fragmentation occurs in skeletal muscle during tumor growth: A link with cancer cachexia? Biochem Biophys Res Commun 270: 533-537, 2000.

24. Ishiko O, Sumi T, Yoshida H, Hyun Y and Ogita S: Angiogenesis in the adipose tissue of tumor-bearing rabbits treated by cyclic plasma perfusion. Int J Oncol 19: 785-790, 2001.

25. Ray SK, Samantaray S, Smith JA, Matzelle DD, Das A and Banik NL: Inhibition of cysteine proteases in acute and chronic spinal cord injury. Neurotherapeutics 8: 180-186, 2011.

26. Ray SK, Matzelle DC, Wilford GG, Hogan EL and Banik NL: E-64-d prevents both calpain upregulation and apoptosis in the lesion and penumbra following spinal cord injury in rats. Brain Res 867: 80-89, 2000

27. Kim KA, Lee YA and Shin MH: Calpain-dependent calpastatin cleavage regulates caspase-3 activation during apoptosis of Jurkat T cells induced by Entamoeba histolytica. Int J Parasitol 37: 1209-1219, 2007 